\title{
ROMANIAN
}

NEUROSURGERY

\author{
Vol. XXXIV | No. 2 June 2020
}

\section{Prognostic factors of ruptured middle cerebral artery aneurysms treated with surgical clipping}

\author{
Samer S. Hoz, \\ Saja A. Albanaa, \\ Ali M. Neamah, \\ Farah W. Abdulmohsin, \\ Zahraa Al-Sharshahi
}




\section{Prognostic factors of ruptured middle cerebral artery aneurysms treated with surgical clipping}

\author{
Samer S. Hoz ${ }^{1}$, Saja A. Albanaa ${ }^{2}$, Ali M. Neamah², \\ Farah W. Abdulmohsin ${ }^{2}$, Zahraa Al-Sharshahi ${ }^{3}$ \\ ${ }^{1}$ Neurosurgeon, Department of Neurosurgery, Neurosurgery \\ Teaching Hospital, Baghdad, IRAQ \\ 2 Medical student, College of Medicine, Baghdad University, \\ Baghdad, IRAQ \\ ${ }^{3} \mathrm{MB}, \mathrm{BCh} B A O(\mathrm{NUI}, \mathrm{RCSI}), \mathrm{LRCP} \& \mathrm{SI}$, Royal College of Surgeons in \\ Ireland (Alumnus), Dublin, IRELAND
}

\section{ABSTRACT}

Background: Spontaneous subarachnoid haemorrhage is an emergent condition that leads to profound morbidity and mortality. It is mainly caused by a ruptured intracranial aneurysm. Herein we described the relationship of different factors and their impact on the outcome of patients who underwent a surgical clipping exclusively to the ruptured middle cerebral artery aneurysm.

Methods: We retrospectively analyzed the medical records of patients admitted to the Neurosurgery Teaching Hospital (NTH) in Baghdad for the period between January 2017-April 2019 of patients who underwent microsurgical clipping for ruptured middle cerebral artery aneurysm. Demographic, clinical, radiological and surgical data were extracted. A univariate analysis was used to illustrate the relationship between the variables and outcome which was assessed using the five scores of the Glasgow Outcome Scale where patients were dichotomized into two groups; favourable (IV + V) and unfavourable (I, II, III).

Results: Within this cohort, a total of 50 patients were studied and the analysis revealed that $92 \%(\mathrm{~N}=46)$ had a favourable outcome and $8 \%(\mathrm{~N}=4)$ had an unfavourable outcome at discharge. The in-hospital mortality was $6 \%(\mathrm{~N}=3)$. Factors that were significantly associated with poor outcome were giant aneurysms, the presence of other unruptured aneurysms, post-operative clinical vasospasm, presence of contralateral weakness, lower pre/post-operative Glasgow coma scores, higher Hunt and Hess, World Federation of Neurosurgical Societies (WFNS) and modified-WFNS grades.

Conclusion: The factors with a significant impact on the outcome of patients with surgically clipped ruptured middle cerebral artery aneurysms were GCS, WFNS, mWFNS, $\mathrm{H} \& \mathrm{H}$, contralateral muscle weakness, size of the aneurysm, presence of other unruptured aneurysms and clinical vasospasm.

\section{INTRODUCTION}

Subarachnoid hemorrhage (SAH) constitutes about $5 \%$ of all strokes. More than $80 \%$ of all Spontaneous SAH is due to ruptured intracranial aneurysms $(7,13)$. CT scan is the best initial test for detecting SAH, CT

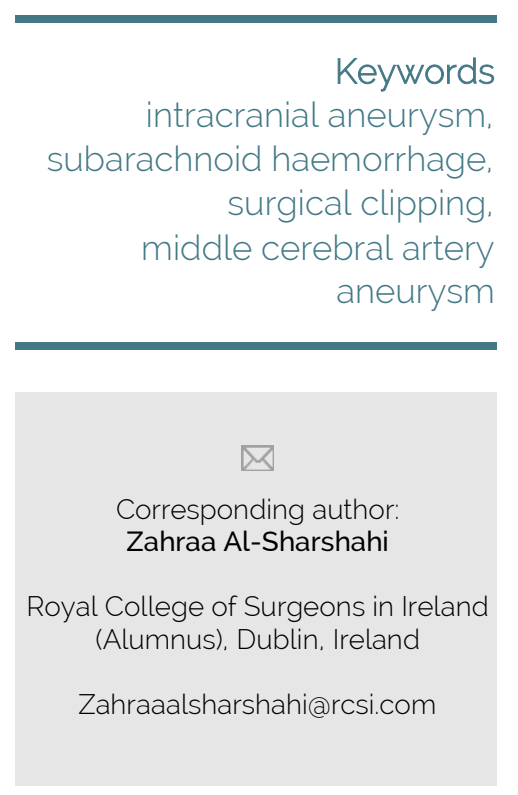

Copyright and usage. This is an Open Access article distributed under the terms of the Creative commons Attribution Non-Commercial No Commons Attribution Non-Commercial No
Derivatives License (https://creativecommons org/licenses/by-nc-nd/4.0/) which permits noncommercial re-use, distribution, and reproduction in any medium, provided the original work is unaltered and is properly cited.

The written permission of the Romanian Society of Neurosurgery must be obtained for commercial re-use or in order to create a derivative work.

ISSN online 2344-4959 (C) Romanian Society of Neurosurgery

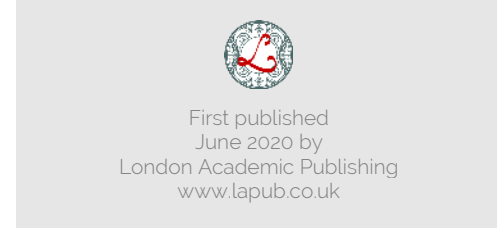


angiography (CTA) is often used to characterize the aneurysm. However, the catheter angiography is the best diagnostic modality to define the aneurysm characteristics preoperatively whenever possible $(10,16)$.

Middle cerebral artery (MCA) aneurysms account for $20 \%$ of all intracranial aneurysms (26). Up to $81 \%$ of MCA aneurysms are located at the bifurcation. In addition, 30\% present with symptoms related to mass effect (14). The MCA aneurysm is the most common aneurysm that co-present with intracerebral hematoma (ICH) (30-50\%) due to its anatomic and hemodynamic features (21). Several grading scales have been used for ruptured SAH including Hunt and Hess $(\mathrm{H} \& H)$, World federation of neurosurgical societies (WFNS) grading and modified WFNS which were reported to be valuable outcome prediction tools $(1,2)$.

Complications of ruptured aneurysms can be categorized as early or late. Such complications include rebleeding, symptomatic vasospasm and hydrocephalus at the rates of $30 \%, 30 \%$ and $20 \%$, respectively (4). Rebleeding is the most serious acute complication and is, at the same time, the most preventable (8). The rate of rebleeding has been declining significantly in association with the establishment of prompt management of SAH by early surgical clipping or endovascular coiling (16).

MCA aneurysm is the most feasible aneurysm for surgical clipping, owing to its accessible location as well as its morphology with a wide neck. Additionally, the MCA aneurysm is unfavorable to be treated with endovascular coiling due to its distal location that renders the difficult $(3,9,15,23)$. In this study, we present a case series which is the first to be reported from Iraq regarding the surgical clipping of ruptured MCA aneurysms.

\section{PATIENTS AND METHODS}

In this retrospective cohort study, data were recruited from 50 patients with $\mathrm{SAH}$ attributed to a ruptured MCA aneurysm. All cases were treated with surgical clipping within 72 hours of aneurysm rupture at Neurosurgery Teaching Hospital in Baghdad/Iraq between January 2017 and April 2019. The inclusion criteria were as follows: age $>18$ years, confirmed diagnosis of aneurysmal SAH by CT-scan and CTA, ruptured MCA aneurysms and surgically treated groups. Cases of traumatic, idiopathic SAHs, unruptured aneurysms and non-MCA aneurysms were excluded from this series. The analyzed parameters included: Age, sex, co-morbidities, reported sudden severe headache as part of the index presentation, pre or post-operative contralateral weakness (MRC grade), seizure, vomiting, neck stiffness, similar previous attacks, clinical vasospasm, pre or post-operative GCS (GCS categorized into; mild $\geq 13$, moderate $12-9$ and severe $\leq 8)$, location and size of the MCA aneurysms, the presence of other unruptured aneurysms, ICH, aneurysmal remnant of post-operative CTA, size of the aneurysm (Giant $>25 \mathrm{~mm}$, Large $11-25 \mathrm{~mm}$, Medium $5-10 \mathrm{~mm}$ and Small $<5 \mathrm{~mm}$ ), the need for temporary clipping, intraoperative rupture, m-WFNS scale, WFNS scale, H\&H scale, Glasgow Outcome Scale at the time of discharge and at six-month follow-up period. At six months follow-up; two cases were lost to follow up in which they were excluded from the analysis.

Fisher exact test was used to evaluate the relationship between the aforementioned factors and GOS at both discharge and six-month intervals. Patients were divided into 2 categorical groups based on the 5 scores of the GOS into favorable (good recovery $\mathrm{V}+$ moderate disability IV) and unfavorable (severe disability III + vegetative state II+ dead I) prognostic groups. The paired data of GOS at discharge and follow-up were also tested for any statistical difference using a Wilcoxon signed-rank test. The correlation between SAH grading scales and GOS was assessed using Kendall's tau correlation coefficient. The level of significance decided on $p$ Value. All statistical analysis was done using commercially available software (SPSS, Version 23.0).

\section{RESULTS}

The study sample included 50 patients, $68 \%(\mathrm{~N}=34)$ of whom were females with a female: male ratio of 2:1. The mean age was $40.32 \pm S D$, with $80 \%(N=30)$ of the females being over 40 years of age and $93.8 \%$ $(\mathrm{N}=15)$ of the males being younger than 40 years of age. Results for patients parameters corresponding to the outcome are shown in tables 1,2 and 3 . Overall, $92 \%(\mathrm{~N}=46)$ of the patients had favorable outcome at discharge (good recovery in $82 \%(\mathrm{~N}=41)$ and moderate disability in $10 \%(\mathrm{~N}=5))$ while $8 \%(\mathrm{~N}=4)$ had unfavorable outcome including $2 \% \quad(\mathrm{~N}=1)$ discharged with severe disability and $6 \%(\mathrm{~N}=3)$ pronounced dead. The mortality was attributed to 
ischemic stroke in two patients and pulmonary embolism in one patient.

The Factors that were associated with unfavorable outcome (GOS scores of I to III) included giant aneurysms, the presence of other unruptured aneurysms, the need for temporary clipping during surgery, the presence of postoperative clinical vasospasm, the presence of pre/post-operative contralateral muscle weakness (Decreased MRC grading), lower GCS scores and higher H\&H, WFNS and m-WFNS scores. In analyzing the correlation between these scales and GOS, m-WFNS had the best negative rank-order correlation coefficient with respect to higher GOS and the overall order was as: m-WFNS then WFNS then H\&H. H\&H showed the least negative correlation with respect to higher GOS (Table 4).

The remaining parameters were not considered as independent risk factors that influence the outcome because they showed no statistical significance. Higher Pre-operative MRC scores were significantly associated with the absence of hematoma ( $P=0.035)$. A higher post-operative MRC grade was notably associated with the absence of post-operative clinical vasospasm $(P<0.001)$. 64\% $(\mathrm{N}=32)$ of patients presented with an $\mathrm{ICH}, 12.5 \%$ $(\mathrm{N}=4)$ of whom had unfavorable outcomes, compared to the patients without ICH where none of them had a poor outcome. The factors that showed statistical significance at discharge have also proven significant at six-month follow-up with the exception of temporary clipping which was not significant at the 6-month follow-up. Temporary clipping which was an infrequent adjunct applied only in $22 \%(\mathrm{~N}=11)$ of all cases for a period of fewer than 10 minutes. $45.5 \%$ of patients in our study with temporary clipping developed vasospasm ( $p=0.017$ ). A Wilcoxon signed-rank test showed that the GOS at discharge differed significantly from the GOS at 6 months follow-up $(Z=-2.33, P=.031)$.

\begin{tabular}{|c|c|c|c|c|c|c|}
\hline \multicolumn{7}{|c|}{ Table 1 Patient's Charecteristics } \\
\hline & & $\begin{array}{c}\text { Favorable } \\
\text { outcome (\%) }\end{array}$ & $\begin{array}{l}\text { Unfavorable } \\
\text { outcome } \\
(\%)\end{array}$ & $\begin{array}{l}\text { Total No. of } \\
\text { cases }\end{array}$ & $\begin{array}{l}P \text { value at } \\
\text { discharge }\end{array}$ & $\begin{array}{l}P \text { value at } \\
\text { follow-up }\end{array}$ \\
\hline \multirow{2}{*}{ Age } & $\leq 40$ & $18(36 \%)$ & $1(2 \%)$ & $19(38 \%)$ & \multirow{2}{*}{1.000} & \multirow{2}{*}{1.000} \\
\hline & $>40$ & $28(56 \%)$ & $3(6 \%)$ & 31 (62\%) & & \\
\hline \multirow{2}{*}{ Sex } & Male & $14(28 \%)$ & $2(4 \%)$ & 16 (32\%) & \multirow{2}{*}{0.584} & \multirow{2}{*}{0.254} \\
\hline & Female & $32(64 \%)$ & $(4 \%) 2$ & $34(86 \%)$ & & \\
\hline \multirow{2}{*}{ Hypertension } & Yes & $15(30 \%)$ & $3(6 \%)$ & $16(32 \%)$ & \multirow{2}{*}{0.127} & \multirow{2}{*}{0.547} \\
\hline & No & $31(62 \%)$ & $1(2 \%)$ & 32 (64\%) & & \\
\hline \multirow{2}{*}{ Diabetes Mellitus } & Yes & $10(20 \%)$ & $2(4 \%)$ & $14(24 \%)$ & \multirow{2}{*}{0.240} & \multirow{2}{*}{0.150} \\
\hline & No & $36(72 \%)$ & $2(4 \%)$ & $38(76 \%)$ & & \\
\hline \multirow{2}{*}{$\begin{array}{l}\text { Sudden severe } \\
\text { headache }\end{array}$} & Yes & $44(88 \%)$ & $3(6 \%)$ & $47(94 \%)$ & \multirow{2}{*}{0.226} & \multirow{2}{*}{0.180} \\
\hline & No & $2(4 \%)$ & $1(2 \%)$ & $3(6 \%)$ & & \\
\hline \multirow{2}{*}{ Vomiting } & Yes & $43(86 \%)$ & $3(6 \%)$ & $46(92 \%)$ & \multirow{2}{*}{0.291} & \multirow{2}{*}{1.000} \\
\hline & No & $3(6 \%)$ & $1(2 \%)$ & $4(8 \%)$ & & \\
\hline
\end{tabular}




\begin{tabular}{|c|c|c|c|c|c|c|}
\hline \multicolumn{7}{|c|}{ Table 1 Patient's Charecteristics } \\
\hline \multirow{2}{*}{ Neck stiffness } & Yes & $44(88 \%)$ & $3(6 \%)$ & $47(94 \%)$ & \multirow{2}{*}{0.226} & \multirow{2}{*}{0.180} \\
\hline & No & $2(4 \%)$ & $1(2 \%)$ & $3(6 \%)$ & & \\
\hline \multirow{2}{*}{$\begin{array}{l}\text { History of } \\
\text { previous attack }\end{array}$} & Yes & $2(4 \%)$ & $1(2 \%)$ & $3(6 \%)$ & \multirow{2}{*}{0.226} & \multirow{2}{*}{0.180} \\
\hline & No & $44(88 \%)$ & $3(6 \%)$ & 47 (94\%) & & \\
\hline \multirow{2}{*}{ Pre-op seizure } & Yes & $8(16 \%)$ & $1(2 \%)$ & $9(18 \%)$ & \multirow{2}{*}{0.560} & \multirow{2}{*}{0.472} \\
\hline & No & $38(76 \%)$ & $3(6 \%)$ & $41(82 \%)$ & & \\
\hline \multirow{2}{*}{ Post-op seizure } & Yes & $4(8 \%)$ & $1(2 \%)$ & $5(10 \%)$ & \multirow{2}{*}{0.353} & \multirow{2}{*}{0.286} \\
\hline & No & $42(84 \%)$ & $3(6 \%)$ & $45(90 \%)$ & & \\
\hline \multirow{2}{*}{$\begin{array}{l}\text { Post-op clinical } \\
\text { vasospasm }\end{array}$} & Yes & $5(10 \%)$ & $4(8 \%)$ & $9(18 \%)$ & \multirow{2}{*}{$0.001 *$} & \multirow{2}{*}{$0.005^{*}$} \\
\hline & No & 41 (82\%) & - & 41 (82\%) & & \\
\hline \multirow{5}{*}{$\begin{array}{l}\text { Pre-op MRC } \\
\text { grading }\end{array}$} & 0 & - & $1(2 \%)$ & $1(2 \%)$ & \multirow{5}{*}{$0.002 *$} & \multirow{5}{*}{$0.004 *$} \\
\hline & 1 & $3(6 \%)$ & $2(4 \%)$ & $5(10 \%)$ & & \\
\hline & 2 & $4(8 \%)$ & $1(2 \%)$ & $5(10 \%)$ & & \\
\hline & 3 & $1(2 \%)$ & - & $1(2 \%)$ & & \\
\hline & Normal & $38(76 \%)$ & - & 38 (76\%) & & \\
\hline \multirow{4}{*}{$\begin{array}{l}\text { Post-op MRC } \\
\text { grading }\end{array}$} & 0 & - & $2(4 \%)$ & $2(4 \%)$ & \multirow{4}{*}{$<0.001$ * } & \multirow{4}{*}{$<0.001$ * } \\
\hline & 1 & - & $1(2 \%)$ & $1(2 \%)$ & & \\
\hline & 3 & $2(4 \%)$ & - & $2(4 \%)$ & & \\
\hline & Normal & $44(88 \%)$ & $1(2 \%)$ & 45 (90\%) & & \\
\hline \multirow{3}{*}{ Initial GCS } & Mild & $42(84 \%)$ & $1(2 \%)$ & 43 (86\%) & \multirow{3}{*}{$<0.001 *$} & \multirow{3}{*}{$<0.001$ * } \\
\hline & Moderate & $4(8 \%)$ & - & $4(8 \%)$ & & \\
\hline & Severe & - & $3(6 \%)$ & $3(6 \%)$ & & \\
\hline \multirow[b]{2}{*}{ Post-op GCS } & Mild & $46(92 \%)$ & $1(2 \%)$ & 47 (94\%) & \multirow[b]{2}{*}{$<0.001$ * } & \multirow[b]{2}{*}{$<0.001 *$} \\
\hline & Severe & - & $3(6 \%)$ & $3(6 \%)$ & & \\
\hline \multicolumn{7}{|c|}{ MRC,medical research council ; GCS,glasgow coma scale ; pre-/post-op, pre-/post-operative } \\
\hline \multicolumn{7}{|c|}{$\begin{array}{l}\text { Significance was determined according to chi-square test. } \\
\text { * Statstically significant }\end{array}$} \\
\hline
\end{tabular}




\begin{tabular}{|c|c|c|c|c|c|c|}
\hline \multicolumn{7}{|c|}{ Table 2 Radiological \& surgical Data } \\
\hline & & $\begin{array}{c}\text { Favorable } \\
\text { outcome (\%) }\end{array}$ & $\begin{array}{c}\text { Unfavorable } \\
\text { outcome } \\
(\%)\end{array}$ & $\begin{array}{l}\text { Total No. of } \\
\text { cases }\end{array}$ & $\begin{array}{l}\text { P value at } \\
\text { discharge }\end{array}$ & $\begin{array}{l}P \text { value at } \\
\text { follow-up }\end{array}$ \\
\hline \multirow{5}{*}{$\begin{array}{l}\text { Aneurysm } \\
\text { location }\end{array}$} & $\begin{array}{l}\text { Right MCA } \\
\text { bifurcation }\end{array}$ & $27(54 \%)$ & $4(8 \%)$ & $31(62 \%)$ & \multirow{5}{*}{0.445} & \multirow{5}{*}{0.624} \\
\hline & $\begin{array}{l}\text { Left MCA } \\
\text { bifurcation }\end{array}$ & $16(32 \%)$ & - & $16(32 \%)$ & & \\
\hline & Right M2 & $1(2 \%)$ & - & $1(2 \%)$ & & \\
\hline & $\begin{array}{c}\text { Right M2 } \\
\text { bifurcation }\end{array}$ & $1(2 \%)$ & - & $1(2 \%)$ & & \\
\hline & Left M2 & $1(2 \%)$ & - & $1(2 \%)$ & & \\
\hline \multirow{4}{*}{ Aneurysm size } & Small & $8(16 \%)$ & $1(2 \%)$ & $9(18 \%)$ & \multirow{4}{*}{$0.004 *$} & \multirow{4}{*}{$0.003 *$} \\
\hline & Medium & $30(60 \%)$ & - & $30(60 \%)$ & & \\
\hline & Large & $7(14 \%)$ & $1(2 \%)$ & $8(16 \%)$ & & \\
\hline & Giant & $1(2 \%)$ & $2(4 \%)$ & $3(6 \%)$ & & \\
\hline \multirow{2}{*}{$\begin{array}{c}\text { Other } \\
\text { unruptured } \\
\text { aneurysm }\end{array}$} & Yes & - & $2(4 \%)$ & $2(4 \%)$ & \multirow[b]{2}{*}{$0.005^{*}$} & \multirow[b]{2}{*}{$0.003^{*}$} \\
\hline & No & $46(92 \%)$ & $2(4 \%)$ & $48(96 \%)$ & & \\
\hline \multirow{2}{*}{$\mathrm{CT}-\mathrm{ICH}$} & Yes & $28(56 \%)$ & $4(8 \%)$ & $32(64 \%)$ & \multirow{2}{*}{0.283} & \multirow{2}{*}{0.543} \\
\hline & No & $18(36 \%)$ & - & $18(36 \%)$ & & \\
\hline \multirow{2}{*}{$\begin{array}{l}\mathrm{ICH} \text { in dominant } \\
\text { hemisphere }\end{array}$} & Yes & $12(37.5 \%)$ & $2(6.3 \%)$ & $14(43.8 \%)$ & \multirow{2}{*}{1000} & \multirow{2}{*}{576} \\
\hline & No & $16(50 \%)$ & $2(6.3 \%)$ & $18(56.3 \%)$ & & \\
\hline \multirow{2}{*}{$\begin{array}{l}\text { Temporary } \\
\text { clipping }\end{array}$} & Yes & $8(16 \%)$ & $3(6 \%)$ & $11(22 \%)$ & \multirow{2}{*}{$0.029 *$} & \multirow{2}{*}{0.127} \\
\hline & No & $38(76 \%)$ & $1(2 \%)$ & 39 (78\%) & & \\
\hline \multirow{2}{*}{ Intra-op rupture } & Yes & $24(48 \%)$ & $3(6 \%)$ & 27 (54\%) & \multirow{2}{*}{0.614} & \multirow{2}{*}{1.000} \\
\hline & No & $22(44 \%)$ & $1(2 \%)$ & $23(46 \%)$ & & \\
\hline \multirow{2}{*}{$\begin{array}{l}\text { Remnant of } \\
\text { Post-op CTA }\end{array}$} & Yes & $1(2 \%)$ & $1(2 \%)$ & $2(4 \%)$ & \multirow{2}{*}{0.155} & \multirow{2}{*}{1.000} \\
\hline & No & 45 (90\%) & $3(6 \%)$ & $48(96 \%)$ & & \\
\hline
\end{tabular}




\begin{tabular}{|c|c|c|c|c|c|}
\hline \multicolumn{6}{|c|}{ Table 2 Radiological \& surgical Data } \\
\hline & $\begin{array}{c}\text { Favorable } \\
\text { outcome (\%) }\end{array}$ & $\begin{array}{l}\text { Unfavorable } \\
\text { outcome } \\
\text { (\%) }\end{array}$ & $\begin{array}{l}\text { Total No. of } \\
\text { cases }\end{array}$ & $\begin{array}{l}P \text { value at } \\
\text { discharge }\end{array}$ & $\begin{array}{l}P \text { value at } \\
\text { follow-up }\end{array}$ \\
\hline \multicolumn{6}{|c|}{$\mathrm{CT}$, computed tomography; ICH, intracerebral hematoma; CTA; computed tomography angiography } \\
\hline \multicolumn{6}{|c|}{ Significance was determined according to chi-square test. } \\
\hline Statistically significant & & & & & \\
\hline
\end{tabular}

\begin{tabular}{|c|c|c|c|c|c|c|}
\hline \multicolumn{7}{|c|}{ Table 3 Neurological grading scales } \\
\hline & & $\begin{array}{c}\text { Favorable } \\
\text { outcome (\%) }\end{array}$ & $\begin{array}{c}\text { Unfavorable } \\
\text { outcome } \\
(\%)\end{array}$ & $\begin{array}{l}\text { Total No. of } \\
\text { cases }\end{array}$ & $\begin{array}{l}P \text { value at } \\
\text { discharge }\end{array}$ & $\begin{array}{l}P \text { value at follow- } \\
\text { up }\end{array}$ \\
\hline \multirow{2}{*}{ Hunt\&Hess } & Level II & $38(76 \%)$ & - & $38(76 \%)$ & \multirow[t]{2}{*}{$0.002^{* *}$} & \multirow[t]{2}{*}{$0.013^{*}$} \\
\hline & Level IV & $8(16 \%)$ & $4(8 \%)$ & $12(24 \%)$ & & \\
\hline \multirow{4}{*}{ WFNS } & I & $37(74 \%)$ & - & $37(74 \%)$ & \multirow{4}{*}{$0.002 *$} & \multirow{4}{*}{$0.006^{*}$} \\
\hline & II & $1(2 \%)$ & - & $1(2 \%)$ & & \\
\hline & III & $4(8 \%)$ & $1(2 \%)$ & $5(10 \%)$ & & \\
\hline & IV & $4(8 \%)$ & $3(6 \%)$ & $7(14 \%)$ & & \\
\hline \multirow{3}{*}{ Modified -WFNS } & I & $37(74 \%)$ & - & $37(74 \%)$ & \multirow{3}{*}{$0.002 *$} & \multirow{3}{*}{$0.003^{*}$} \\
\hline & II & $5(10 \%)$ & $1(2 \%)$ & $6(12 \%)$ & & \\
\hline & IV & $4(8 \%)$ & $3(6 \%)$ & $7(14 \%)$ & & \\
\hline \multicolumn{7}{|c|}{ WFNS; world federation of neurosurgical societies } \\
\hline \multicolumn{7}{|c|}{ Significance was determined according to chi-square test. } \\
\hline
\end{tabular}


Table 4 Rank-order correlation coefficients of different grading systems on higher GOS

\begin{tabular}{|c|c|c|}
\hline Rank order Correlation coefficient & P value \\
\hline modified-WFNS & -0.56 & $<0.001$ \\
\hline WFNS & -0.55 & $<0.001$ \\
\hline Hunt\&Hess & -0.54 & $<0.001$ \\
\hline WFNS; world federation of neurosurgical societies,
\end{tabular}

\section{DisCUSSION}

Aneurysmal SAH is one of the most serious and often deadly phenomena (12). MCA aneurysm is one of the commonest causes of aneurysmal SAH (6). It is commonly located at the bifurcation and constitutes $81 \%$ of all unruptured MCA aneurysm and $87 \%$ of the ruptured ones (24).

Patient's characteristics: Our data showed a female predominance with a female: male ratio of 2:1. Although there appears to be a tendency towards a more favorable outcome in both females and younger patients, this failed to achieve statistical significance in our study; a finding that was also reported by similar studies $(11,17,24)$. The presence of co-morbidities is often cited as a significant risk factor for aneurysm rupture, but their prognostic significance is still uncertain $(14,25)$. In our data, concomitant diseases, history of previous attacks and seizures were not found to be significantly associated with an unfavorable outcome.

Vasospasm is classified as one of the major complications associated with MCA aneurysm clipping (26). In this study, we exclusively evaluated post-operative clinical vasospasm which was defined as a deterioration in the neurologic status (speech or motor) along with the presence of new ischemic CT changes or CT-angiography-defined vessels spasm. Vasospasm was only evident in $18 \%(\mathrm{~N}=9)$ of patients and was noted to be associated with worse outcomes. Motor weakness is also a known neurological complication that follows aneurysmal SAH with an incidence of $14-29 \%$ (12). Hereby, we evaluated the presence of contralateral limb weakness both pre and post-operatively, using the MRC grading score. All patients showed a more marked weakness in the upper limbs compared to the lower limbs which reflects the anatomical territory supplied by the MCA. Patients with Pre or post-operative MRC grading scores for muscle power of $(0,1)$ showed a significant tendency toward a poorer outcome and those with no weakness showed a significant tendency toward a better prognosis. Additionally, higher pre-operative MRC scores were associated with the absence of hematoma whereas post-operative MRC was associated with postoperative clinical vasospasm. These findings were consistent with other studies reporting vasospasm and cerebral ischemia as the most common mechanisms that may lead to motor weakness in aneurysmal SAH (12).

Cerebral vasospasm is directly linked to cerebral ischemia and patients may present with stroke-like symptoms including motor weakness, hence low MRC scores are not uncommon in patients with cerebral vasospasm. The presence of lower (severe) pre or post-operative GCS scores was found to be significantly associated with a lower GOS in contrast to the higher (mild) GCS scores which were significantly associated with higher GOS.

Radiological and aneurysmal characteristics: Aneurysm characteristics such as aneurysm location, size and the presence of other unruptured aneurysms are important factors that impact the prognosis of ruptured cranial aneurysms $(14,25)$. Most reports inversely correlate aneurysmal size with the outcome (11). Our data showed that giant aneurysms were associated with unfavorable outcome whereas medium-sized aneurysms were associated with a more favorable outcome. Notably, Ruggeri et al reported that the diameter of the 
aneurysm didn't significantly affect the outcome which is in contrast to what most reports (19). Our study took the side of the aneurysm (right vs left) into account. $62 \%$ of the aneurysms were located on the right MCA bifurcation and were noted to be associated with unfavorable outcomes; this observation did not, however, achieve statistical significance. This can be attributed to the fact that most of the cases in our sample are right-handed, with a left dominant hemisphere. However, Brawanski et al reported that the side of the aneurysm did not affect the outcome directly, but rather determined the side of infarction (5). In addition to the aneurysm size and location, the presence of other unruptured aneurysms was found to significantly associated with an unfavorable outcome $(P=0.005)$. This finding is in contrast to the study performed by Rodriguez-Hernandez et al who stated that the number of aneurysms did not impact the outcome; although this series did not take into account the status of the aneurysm whether it was ruptured or not (25). MCA aneurysm is known to be the most common intracranial aneurysm that is associated with an ICH (24).In a paper by Shimoda et al which studied 47 patients presented with ruptured MCA aneurysm associated with a hematoma, it was found that $42 \%(\mathrm{~N}=20)$ had an $\mathrm{ICH}$ in the dominant hemisphere that led to a poor outcome but was reported to be non-significant similarly to the findings of this cohort (22).

Surgical parameters: Intra-operative rupture was reported as a factor that doesn't impact the surgical outcome (20). This finding is consistent with our results where intra-operative rupture showed a nonsignificant trend toward a poorer outcome. The application of temporary clipping was significantly associated with lower GOS at discharge but not at follow-up. This can be attributed to the fact that temporary clipping was noted to increase the risk of postoperative clinical vasospasm significantly where $45.5 \%$ of patients in our study with temporary clipping developed vasospasm ( $p=0.017$ ). Therefore, temporary clipping was found to be a dependent factor regarding follow-up. We observed that the absence of post-operative CTA remnants led to a favorable outcome but this was not statistically significant.

Grading scales: WFNS, mWFNS, and $\mathrm{H} \& \mathrm{H}$ are commonly used neurological scales that aid in surgical decision and predict the outcome of patients with aneurysmal SAH (1).H\&H scale showed the least negative correlation in respect to higher GOS since it relies only on general subjective terms that depend on the judgment and interpretation, leading to a blurring of the lines between grades which in return would increase the inter-rater reliability $(2,18)$. Therefore, this scale is more useful in the selection of surgical candidates than outcome prediction. WFNS scale is superior to H\&H as it depends on both GCS and the absence/presence of neurologic deficits. When both WFNS and m-WFNS were compared in terms of correlation with GOS, m-WFNS correlated slightly stronger. Regarding m-WFNS, none of the cases in our study scored grade III, $12 \%(\mathrm{~N}=6)$ scored grade II and the rest scored grade I and IV. Hence grades II and III are the only different grades between the 2 scales, explaining the slight preference of m-WFNS over WFNS (Table 4).

Limitations: First, this is a retrospective cohort from a single neurosurgical center with a small sample size that only included univariate analysis, making the conclusions non-generalizable. Second, the absence of intravascular coiling facilities made surgical clipping the only available treatment option in Iraq, limiting our ability in comparing the results to those of intravascular coiling.

\section{CONCLUSION}

Factors that may predict poorer outcomes in ruptured MCA aneurysm Clippinginclude: Higher mWFNS, WFNS, and H\&H scores, lower GCS scores, the presence of clinical vasospasm, the presence of hemiparesis, the presence of other unruptured aneurysms and aneurysm size (giant aneurysms). $m$ WFNS was found to be slightly superior to the other neurologic scales in predicting the outcome.

\section{CONFLICTS OF INTEREST}

The authors declare no conflict of interests

\section{ABBREVIATIONS}

CT: Computerized Tomography

MCA: middle cerebral artery

$\mathrm{SAH}$ : subarachnoid haemorrhage

$\mathrm{H}$ and $\mathrm{H}$ : Hunt and Hess scale

WFNS: World Federation of Neurosurgical Societies scale

\section{REFERENCES}

1. Adams Jr HP. Clinical scales to assess patients with stroke. InStroke 2011 Jan 1 (pp. 307-333). WB Saunders. 
2. Aggarwal A, Dhandapani S, Praneeth $K$, Sodhi HB, Pal SS, Gaudihalli S, Khandelwal N, Mukherjee KK, Tewari MK, Gupta SK, Mathuriya SN. Comparative evaluation of H\&H and WFNS grading scales with modified $\mathrm{H} \& \mathrm{H}$ (sans systemic disease): A study on 1000 patients with subarachnoid hemorrhage. Neurosurgical review. 2018 Jan 1;41(1):241-7.

3. Alreshidi M, Cote DJ, Dasenbrock HH, Acosta M, Can A, Doucette J, Simjian T, Hulou MM, Wheeler LA, Huang K, Zaidi HA. Coiling versus microsurgical clipping in the treatment of unruptured middle cerebral artery aneurysms: a meta-analysis. Neurosurgery. 2018 Feb 9;83(5):879-89.

4. Bakker NA, Metzemaekers JD, Groen RJ, Mooij JJ, Van Dijk JM. International subarachnoid aneurysm trial 2009: endovascular coiling of ruptured intracranial aneurysms has no significant advantage over neurosurgical clipping. Neurosurgery. 2010 May 1;66(5):961-2.

5. Brawanski N, Kashefiolasl S, Won SY, Tritt S, Berkefeld J, Senft C, Seifert V, Konczalla J. Does aneurysm side influence the infarction side and patients' outcome after subarachnoid hemorrhage?. PloS one. 2019;14(11).

6. Chyatte D, Porterfield R. Nuances of middle cerebral artery aneurysm microsurgery. Neurosurgery. 2001 Feb 1;48(2):339-46.

7. Dabilgou AA, Drave A, Kyelem JM, Naon L, Napon C, Kabore J. Spontaneous Subarachnoid Haemorrhage in Neurological Setting in Burkina Faso: Clinical Profile, Causes, and Mortality Risk Factors. Neurology research international. 2019;2019.

8. Danière $F$, Gascou $G$, de Champfleur NM, Machi $P$, Leboucq N, Riquelme C, Ruiz C, Bonafé A, Costalat V. Complications and follow up of subarachnoid hemorrhages. Diagnostic and interventional imaging. 2015 Jul 1;96(7-8):677-86.

9. de Gans K, Nieuwkamp DJ, Rinkel GJ, Algra A. Timing of aneurysm surgery in subarachnoid hemorrhage: a systemic review of the literature. Neurosurgery. 2002;50:336-40.

10. Đilvesi $Đ$, Cigić T, Papić V, Horvat I, Karan M, Vuleković P. The Fisher grade in predicting a degree of cerebral vasospasm in patients after intracranial aneurysm rupture. Vojnosanitetski pregled. 2016;73(4):349-52.

11. Hamdan A, Barnes J, Mitchell P. Subarachnoid hemorrhage and the female sex: analysis of risk factors, aneurysm characteristics, and outcomes. Journal of neurosurgery. 2014 Dec 1;121(6):1367-73.

12. Jang SH, Do Lee H. The pathogenic mechanisms of motor weakness following aneurysmal subarachnoid hemorrhage: A review. Neurology Asia. 2017 Sep 1;22(3).

13. Javadpour $M$, Silver $N$. Subarachnoid haemorrhage (spontaneous aneurysmal). BMJ clinical evidence. 2009;2009.

14. Oh JW, Lee JY, Lee MS, Jung HH, Whang K, Brain Research
Group. The meaning of the prognostic factors in ruptured middle cerebral artery aneurysm with intracerebral hemorrhage. Journal of Korean Neurosurgical Society. 2012 Aug;52(2):80.

15. Qin S, Sun X, Hui P, Li J, Wang Z. Analysis on prognostic factors of patients with ruptured intracranial aneurysms. Int J Clin Exp Med. 2017 Jan 1;10(1):1303-8.

16. Rahmanian A, Derakhshan N, Alibai EA. Outcome of InHospital Rebleeding and Early Aneurysm Rupture at the Referral Center. Iranian Journal of Neurosurgery. 2018 Jul 15;4(2):93-100.

17. Rodríguez-Hernández A, Sughrue ME, Akhavan $S$, Habdank-Kolaczkowski J, Lawton MT. Current management of middle cerebral artery aneurysms: surgical results with a "clip first" policy. Neurosurgery. 2012 Nov 30;72(3):415-27.

18. Rosen DS, Macdonald RL. Subarachnoid hemorrhage grading scales. Neurocritical care. 2005 Apr 1;2(2):110-8.

19. Ruggeri AG. Ruptured Middle Cerebral Artery Aneurysms: Retrospective Study and Multivariate Analysis of 105 Patients Treated by Surgical Clipping. Journal of Neurosurgery Research and Reviews. 2017 Nov 25;1(1).

20. Sandalcioglu IE, Schoch B, Regel JP, Wanke I, Gasser T, Forsting M, Stolke D, Wiedemayer H. Does intraoperative aneurysm rupture influence outcome? Analysis of 169 patients. Clinical neurology and neurosurgery. 2004 Mar 1;106(2):88-92.

21. Santiago-Dieppa DR, Pannell JS, Khalessi AA. Endovascular and surgical options for ruptured middle cerebral artery aneurysms: review of the literature. Stroke research and treatment. 2014;2014.

22. Shimoda M, Oda S, Mamata Y, Tsugane R, Sato O. Surgical indications in patients with an intracerebral hemorrhage due to ruptured middle cerebral artery aneurysm. Journal of neurosurgery. 1997 Aug 1;87(2):170-5.

23. Taha MM, Alawamry A, Abdelbary TH. Outcome of microsurgical clipping of anterior circulation aneurysms during the period of vasospasm: single center experience in Egypt. Egyptian Journal of Neurosurgery. 2019 Dec $1 ; 34(1): 5$

24. Ulm AJ, Fautheree GL, Tanriover N, Russo A, Albanese E, Rhoton Jr AL, Mericle RA, Lewis SB. Microsurgical and angiographic anatomy of middle cerebral artery aneurysms: prevalence and significance of early branch aneurysms. Operative Neurosurgery. 2008 May 1;62(suppl_5):ONS344-53.

25. Wang GX, Yu JY, Wen L, Zhang L, Mou KJ, Zhang D. Risk factors for the rupture of middle cerebral artery bifurcation aneurysms using CT angiography. PLoS One. 2016 Dec 15;11(12):e0166654.

26. Yang $\mathrm{W}$, Huang J. Treatment of middle cerebral artery (MCA) aneurysms: a review of the literature. Chinese Neurosurgical Journal. 2015 Dec;1(1):1. 